\title{
Novel association of RP1 gene mutations with autosomal recessive retinitis pigmentosa
}

\author{
S Khaliq, A Abid, M Ismail, A Hameed, A Mohyuddin, P Lall, A Aziz, K Anwar, S Q Mehdi
}

J Med Genet 2005;42:436-438. doi: 10.1136/jimg.2004.024281

$\mathrm{R}$ etinitis pigmentosa $(\mathrm{RP})$ is the most prevalent hereditary retinal degenerative disease. To date, approximately 40 loci and mutations in more than 25 genes have been identified as the cause of various types of RP. ${ }^{1}$ The gene for human oxygen regulated photoreceptor protein (RPI) encodes a protein of 2156 amino acids that is localised in the connecting cilia of both rod and cone photoreceptors. ${ }^{2}$ The RPl protein is required for the morphogenesis of the outer segments of the photoreceptor cells. ${ }^{3}$ Several laboratories have found mutations in the $R P I$ gene to be the cause of autosomal dominant retinitis pigmentosa (adRP). ${ }^{5-7}$ However, to our knowledge, association of the RPl gene with recessive RP has never been reported.

The aim of the present study was to map the disease locus for three consanguineous Pakistani families suffering from $\mathrm{RP}$. We present mapping of these autosomal recessive RP families to the $8 \mathrm{ql} 1$ locus. The results show, for the first time, that in these families a form of arRP is caused by homozygous mutations of the RPI gene. Although these mutations were found in the parents and some of the siblings who had normal vision (carriers) in a heterozygous state, they were not found in any of a panel of 100 normal controls.

\section{PATIENTS AND METHODS}

We studied three consanguineous Pakistani families (442RP, $452 \mathrm{RP}$, and 336RP) suffering from autosomal recessive RP (fig 1). The patients had night blindness since early childhood and progressive deterioration of vision with age. All the patients were completely blind by the age of $12-15$ years in the case of the 442RP and 452RP families and 17-18 years in the 336RP family. Fundoscopic examination and electroretinographic (ERG) analyses were carried out on all the patients, their heterozygous parents and siblings, and unaffected normal siblings.

Fundoscopic examination of the affected individuals, V:3 (22 years), V:5 ( 15 years), and V:12 ( 18 years) from the 442RP family and IV:1 (25 years), IV:6 (12 years), and IV:9 ( 18 years) from the 452RP family, was carried out. All the patients had attenuation of blood vessels, pale optic disc, and stippling of macula with no bony spicules. Parents (carriers) from all branches of these families and some of the normal siblings were also examined. None of the (normal) individuals had any sign of RP. Electroretinographic (ERG) examination showed non-recordable or reduced ERG response in all the patients. Their unaffected (heterozygous/ carriers) parents (age range 35-60 years) had normal ERGs (rod and cone function). Clinical examination of patients from 336RP family also revealed typical features of RP, including pigment deposition, attenuation of retinal blood vessels, and pale optic disc. Patients IV:2 and IV:4 (aged 17 years and 22 years, respectively) had pigment deposition in both the equatorial and macular regions at the time of investigation. Fundoscopic examination of the unaffected heterozygous father (40 years) showed neither pigment deposition nor abnormality of the retina.

\section{Key points}

- RPl gene mutations are the second most common cause of autosomal dominant retinitis pigmentosa (RP).

- A homozygous T373I mutation (ACA to ATA) was found to segregate with the disease in two autosomal recessive (ar) RP families from Pakistan.

- A 4 bp insertion at nucleotide 1461 adds a termination codon after codon 487 in another arRP family. This results in the production of a severely truncated protein of 487 instead of 2156 amino acids.

- A second missense A669T substitution (GCC to ACC) was found in a heterozygous state in one sample from a panel of 150 RP patients.

- These results suggest that the frequency of RP1 gene mutations is relatively low in Pakistan.

- To our knowledge this study provides the first report of the involvement of mutations in the RPI gene in the autosomal recessive RP phenotype.

For genetic analysis, peripheral blood samples were collected from all members of the families with informed consent. Control samples were also collected from 100 ethnically matched, unrelated, normal Pakistani individuals. Patients were examined at the retina clinic of one of the KRL General Hospital, Islamabad, the Al-Shifa Trust Eye Hospital, Rawalpindi, and the Christian Hospital, Taxila.

\section{Linkage analysis}

Genomic DNA was extracted from whole blood using the standard phenol/chloroform extraction procedure. DNA was amplified using specific primers for polymorphic microsatellite markers (version 8; Research Genetics) for known genes and loci associated with various types of retinal degeneration according to the conditions described previously. ${ }^{8}$

\section{Mutation detection}

Exon specific primers were designed from the genomic sequence of $R P I .^{7}$ PCR was performed in a $50 \mu$ l reaction volume using $1 \mu \mathrm{g}$ of genomic DNA. Heteroduplex analysis was performed using an automated denaturing high performance liquid chromatography (DHPLC) instrument (WAVE DNA Fragment Analysis System, Transgenomic, Crewe, UK). ${ }^{9}$ Samples were prepared by denaturing and re-annealing of the unpurified PCR products. The temperature conditions required for the successful resolution of heteroduplexes were obtained from the Stanford University website (http:// insertion.stanford.edu/melt.html).

Following heteroduplex analysis, DNA from all family members was sequenced in the forward and reverse directions using a commercially available kit (Big Dye, Applied Biosystems), and the products were analysed on an ABI 

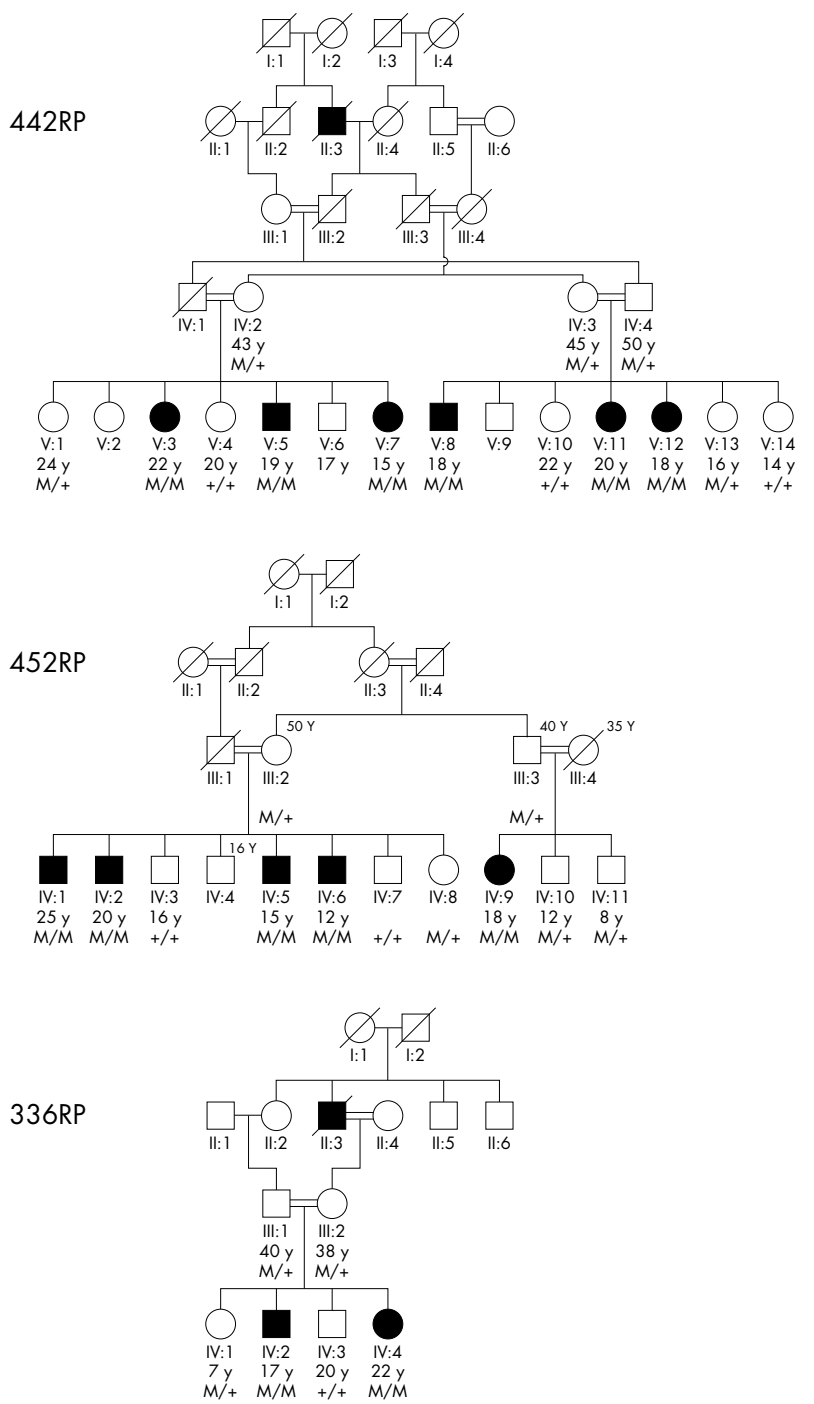

Figure 1 Pedigrees of autosomal recessive RP families 442RP, 452RP, and 336RP. Normal and mutated alleles for each individual are labelled $(+)$ and $(M)$ respectively.

Prism 377 automated DNA sequencer. To examine the possibility that the mutations are polymorphisms, a panel of 100 normal individuals was also analysed for RPI gene mutations, initially by DHPLC followed by direct DNA sequencing.

\section{RESULTS AND DISCUSSION}

Exclusion studies of known RP loci were carried out for the three arRP families. Homozygosity was observed for markers D8S285 and D8S1815 for all the patients. This locus contains $R P 1$, which has previously been reported to be associated with dominant RP.

As most of the mutations identified previously are clustered in a small region, the $5^{\prime}$ region of exon 4 was screened..$^{10}$ DHPLC analysis showed heteroduplex peaks for the unaffected parents and some of the siblings of the patients in both the 442RP and 452RP families. Samples of all affected individuals showed homoduplexes. All the family members were then sequenced to identify the change in exon 4. Sequencing analysis revealed that patients had a homozygous $\mathrm{C} \rightarrow \mathrm{T}$ substitution at nucleotide 1118 (fig $2 \mathrm{~A}$ ). Parents of the affected individuals were heterozygous for this change. This substitution causes a missense codon 373 that codes for isoleucine (ATA) instead of threonine (ACA) in the mutated protein product. The normal family members including their parents (carriers) who are heterozygous for the Thr373Ile change have normal vision with no signs of RP. Eukaryotic linear motif (ELM; http://elm.eu.org/) searches showed that the T373I missense mutation abolishes the glycogen synthase phosphorylation recognition site (GSK3), resulting in a conformational change in the mutated protein. To check if there was another mutation in this gene associated with the disease phenotype in both these families, the remaining three coding exons of the RPI gene were also sequenced. No other disease associated change was found in any patient of the $442 \mathrm{RP}$ and 452RP families. None of the 100 normal controls carried the T373I change in the homozygous state, although some heterozygous individuals were observed. Payne et al ${ }^{7}$ have reported this change to cause adRP; however, Berson et $a l^{11}$ categorised this mutation as non-pathogenic because it did not cosegregate with the disease phenotype in one of their adRP families. They suggested that mutations in $R P I$ might be recessive, causing RP or some other retinal disease. Our data shows that the homozygous T373I change causes arRP in consanguineous families.

In the unaffected members of the third family (336RP), heteroduplex peaks were also observed for another fragment of exon 4. Sequencing analysis of all family members showed a homozygous $4 \mathrm{bp}$ insertion (1461-1465insTGAA) in the patients (fig 2B). This insertion adds a stop codon immediately after nucleotide 1461, resulting in a severely truncated protein of 487 instead of 2156 amino acids. This $4 \mathrm{bp}$ insertion was present in a heterozygous state in some unaffected members of the family, including the parents of the patients. Subsequently, the panel of normal controls was screened for this insertion. None of the 100 normal individuals carried this change.

A random panel of $150 \mathrm{RP}$ patients was also screened for RPI gene mutations. A heterozygous single base pair $\mathrm{G} \rightarrow \mathrm{A}$ substitution at nucleotide position 2005 was found in one patient (fig 2C). This substitution replaces alanine (GCC) with threonine (ACC) at codon 669. This change was not found in any of the 100 normal individuals.

In addition to these disease causing mutations, several sequence variants and polymorphisms were also found in this study (table 1). These results show that two polymorphisms, N985Y and P1205P, are prevalent in the Pakistani samples.

Previous studies have identified 21 different disease causing mutations that are responsible for $6-10 \%$ cases of adRP in ethnically diverse populations..$^{511}$ None of these mutations were found in the Pakistani samples. Identification of only one mutation in the unrelated $150 \mathrm{RP}$ patients and two mutations with recessive RP among a large number of patients and families shows that RPI gene mutations are not a major cause of RP in Pakistan.

Dominant RP caused by mutations in the RPl gene often shows late onset of the disease phenotype, usually by the third decade of life. Pierce et al ${ }^{6}$ observed that patients suffering from adRP who were heterozygous for the RPl mutation had classic, less severe adRP phenotype with late onset of disease. In contrast, patients that were homozygous for the mutation in the same family had substantially more severe forms of the disease and the age of onset was variable. ${ }^{12}$ All three recessive Pakistani families presented here showed the severe form of RP with early onset.

It is well known that Mendelian disorders caused by the dysfunction of a single gene have a wide heterogeneity of disease phenotypes. ${ }^{13} \mathrm{RP}$ is a heterogeneous group of retinal disorders and mutations within a single gene are known to cause different clinical phenotypes. ${ }^{14-17}$ The work presented here shows, for the first time, that arRP is also caused by (homozygous) mutations in the RPl gene. This could be due 


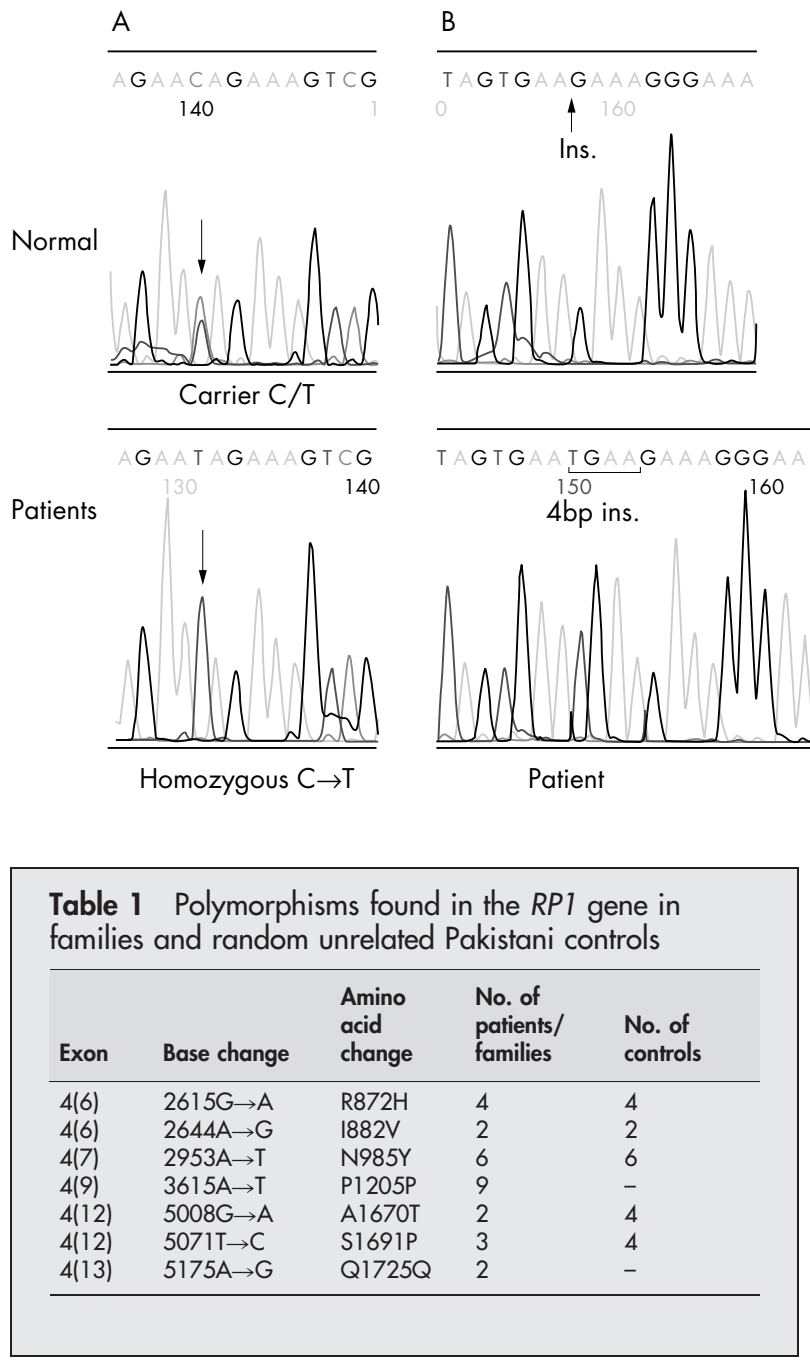

to centuries of consanguineous marriages in this gene pool, altering the penetrance of the mutated gene in the heterozygous state.

\section{ACKNOWLEDGEMENTS}

The authors thank the family members for taking part in this study. This work was supported by Wellcome Trust grant number 063406/Z/ 2000/Z to S Q Mehdi.

\section{Authors' affiliations}

S Khaliq, A Abid, M Ismail, A Hameed, A Mohyuddin, K Anwar, S Q Mehdi, Dr. A. Q. Khan Research Laboratories, Biomedical and Genetic Engineering Division, Islamabad, Pakistan. GPO Box 2891, 24 Mauve Area, Islamabad, Pakistan; sqmehdi@comsats.net.pk P Lall, Christian Hospital, Faisal Shaheed Road, Taxila, Pakistan A Aziz, Al-Shifa Trust Eye Hospital, Rawalpindi, Pakistan Competing interests: none declared

Correspondence to: Dr S Qasim Mehdi, Biomedical and Genetic Engineering Division, Dr A Q Khan Research Laboratories, GPO Box 2891, 24 Mauve area, Islamabad, Pakistan; sqmehdi@comsats.net.pk

Received 21 June 2004

Revised 3 August 2004

Accepted 24 August 2004

\section{REFERENCES}

1 University of Texas-Houston Health Science Center. RetNet. Available at: http://www.sph.uth.tmc.edu/Retnet/disease.htm.

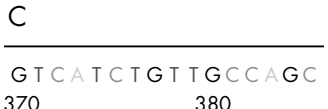

Figure 2 Top electropherograms are of normal/carrier sequences. Bottom shows (A) a homozygous $\mathrm{C} \rightarrow \mathrm{T}$ substitution at nucleotide 1118 (Thr373lle) in patients from families $442 \mathrm{RP}$ and 452RP, (B) a homozygous 4 bp insertion introducing a stop codon at nucleotide 1461 in patients from the 336RP family, and (C) a heterozygous $\mathrm{G} \rightarrow \mathrm{A}$ mutation at nucleotide 2005

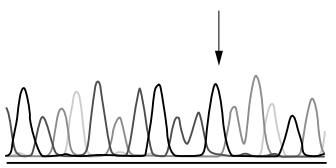
(Ala $\rightarrow$ Thr) in a single individual from a panel of 150 random RP patients.
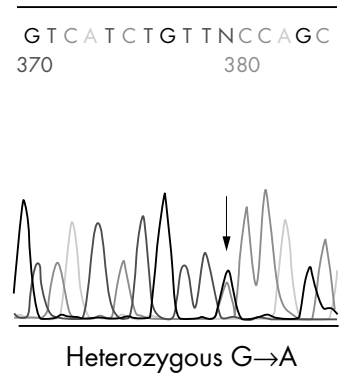

2 Liu Q, Zhou J, Daiger SP, Farber DB, Heckenlively JR, Smith JE, Sullivan LS, Zuo J, Milam AH, Pierce EA. Identification and sub cellular localization of the RP1 protein in human and mouse photoreceptors. Invest Opthalmol Vis Sci 2002;43:22-32.

3 Gao J, Cheon K, Nusinowitz S, Liu Q, Di Bei, Atkins K, Azimi A, Daiger SP, Farber DB, Heckenlively JR, Pierce EA, Sullivan LS, Zuo J. Progressive photoreceptor degeneration, outer segment dysplasia, and rhodopsin mislocalization in mice with targeted disruption of the ritinitis pigmentosa- 1 (RP1) gene. PNAS 2002;99:5698-703.

$4 \mathrm{RP1}$ is required for the correct stacking of the outer segment discs. Invest Opthalmol Vis Sci 2003:44:4171-83.

5 Bowne SJ, Daiger SP, HimsMM, Sohocki MM, Malone KA, McKie AB Heckenlively JR, Birch DG, Inglehearn CF, Bhattacharya SS, Bird A, Sullivan LS. Mutations in the RPI gene causing autosomal dominant retinitis pigmentosa. Hum Mol Genet 1999;8:2121-8.

6 Pierce EA, Quinn T, Meehan T, McGee TL, Berson EL, Dryja TP. Mutations in a gene encoding a new oxygen-regulated photoreceptor protein cause dominant retinitis pigmentosa. Nat Genet 1999;22:248-54.

7 Payne A, Vithana E, Khaliq S, Hameed A, Deller J, Abu-Safieh L, Kermani S, Leroy BP, Mehdi SQ, Moore AT, Bird AC, Bhattacharya SS. RP1 protein truncating mutations predominate at the RP1 adRP locus. Invest Ophth Vis Sci 2000;41:4069-73.

8 Khaliq S, Abid A, Hameed A, Anwar K, Mohyuddin A, Azmat Z, Shami SA, Ismail M, Mehdi SQ. Mutation screening of Pakistani families with congenital eye disorders. Exp Eye Res 2003;76:343-8.

9 Underhill PA, Shen P, Lin AA, Jin L, Passarino G, Yang WH, Kauffman E, Bonne-Tamir B, Bertranpetit J, Francalacci P, Ibrahim M, Jenkins T, Kidd JR, Mehdi SQ, Seielstad MT, Wells RS, Piazza A, Davis RW, Feldman MW Cavalli-Sforza LL, Oefner J. Y chromosome sequence variation and the history of human populations. Nat Genet 2000;26:358-61.

10 Dietrich K, Jacobi FK, Tippmann S, Schmid R, Zrenner E, Wissinger B, Apfelstedt-Sylla E. A novel mutation of the RP1 gene (Lys778ter) associated with autosomal dominant retinitis pigmentosa. $\mathrm{Br} J$ Ophthalmol 2002;86:328-32

11 Berson EL, Grimsby JL, Adams SM, McGee TL, Sweklo E, Pierce EA, Sandberg MA, Dryja TP. Clinical features and mutations in patients with dominant retinitis pigmentosa-1 (RP1). Invest Opthalmol Vis Sci 2001;42:2217-24.

12 Sullivan LS, Heckenlively JR, Bowne SJ, Zuo J, Hide WA, Gal A, Denton M, Inglehearn CF, Blanton SH, Daiger SP. Mutations in a novel retina-specific gene cause autosomal dominant retinitis pigmentosa. Nat Genet 1999:22:255-9.

13 Bell J. Predicting disease using genomics. Nature 2004;429:453-6.

14 Rosenfeld PJ, Cowley GS, McGee TL, Sandberg MA, Berson EL, Dryja TP. A null mutation in the rhodopsin gene causes rod photoreceptor dysfunction and autosomal recessive retinitis pigmentosa. Nat Genet 1992;1:209-13.

15 Dryja TP, McGee TL, Reichel E, Hahn LB, Cowley GS, Yandell DW, Sandberg MA, Berson EL. A point mutation of the rhodopsin gene in one form of retinitis pigmentosa. Nature 1990;343:364-6.

16 Gerber S, Perrault I, Hanein S, Barbet F, Ducroq D, Ghazi I, MartinCoignard D, Leowski C, Homfray T, Dufier JL, Munnich A, Kaplan J, Rozet JM. Complete exon intron structure of the RPGR-interacting protein (RPGRIP1) gene allows the identification of mutations underlying Leber congenital amaurosis. Eur J Hum Genet $2001 ; 9: 561-71$.

17 Hameed A, Abid A, Aziz A, Ismail M, Mehdi SQ, Khaliq S. Evidence of RPGRIP1 gene mutations associated with recessive cone-rod dystrophy. J Med Genet 2002;40:616-19. 